Pacific

Journal of

Mathematics

EVERY TIGHT IMMERSION IN THREE-SPACE OF THE PROJECTIVE PLANE WITH ONE HANDLE IS ASYMMETRIC

Davide P. Cervone

Volume 215 No. 2

June 2004 


\title{
EVERY TIGHT IMMERSION IN THREE-SPACE OF THE PROJECTIVE PLANE WITH ONE HANDLE IS ASYMMETRIC
}

\author{
Davide P. Cervone
}

\begin{abstract}
Kuiper's original analysis of tight surfaces showed that every surface has a tight immersion in three-space except for the Klein bottle and the projective plane, which have none, and the projective plane with one handle, for which he was unable to determine whether a tight immersion was possible. The latter obtained a unique position among surfaces when it was shown that no smooth tight immersion of it can be formed, while a polyhedral one does exist. Continuing in its role as an unusual example, this surface has another unexpected property, demonstrated here: Any tight immersion is necessarily asymmetric, while every other surface can be immersed tightly and symmetrically in space.
\end{abstract}

\section{Introduction}

The real projective plane with one handle has proven to be an unusual surface in more than one way. Initially, when Kuiper determined which surfaces admit tight immersions in space $([\mathbf{1 3}],[\mathbf{1 4}]$ and $[\mathbf{1 5}])$, it was the sole surface for which the answer was unknown. This situation persisted for thirty years until two important results appeared in rapid succession. First, in 1992, Haab showed [10] that no smooth tight immersion of the real projective plane with one handle is possible. The methods he used rely heavily on the smoothness of the immersion, and so the question remained open for polyhedral surfaces. Two years later, in an unexpected result [8], the author produced a polyhedral example of a tight immersion of this surface, representing one of only a handful of low-dimensional examples where the polyhedral and smooth theories differ in a significant way.

The reason why a polyhedral immersion exists while no smooth one does is not well understood. In [7] the author shows that the obstruction to smoothing the polyhedral example is not a local one, but further study is still warranted. In an attempt to understand this surface better, the author began a search for a more symmetric tight immersion, but was unable to produce one. As it turns out, there is no symmetric tight immersion, which is the main result of this paper. 
Theorem 1.1. Any tight immersion of the real projective plane with one handle in three-space is necessarily asymmetric.

The proof begins in Section 4, and is broken into several parts. The reflections and rotation-reflections are ruled out easily, leaving only rotations as possible symmetries for this surface. The cases of an $n$-fold symmetry with $n>2$ and $n=2$ are analyzed separately. Section 4.1 shows that, in a tight immersion with $n$-fold symmetry where $n \geq 3$, the central core of the surface can be used to make an immersion of the projective plane having exactly one maximum, one minimum and one saddle; but in Section 4.2, a close study of the requirements for the central critical level show that no such symmetric projective plane exists. Finally, in Section 4.4, we prove that no closed surface of odd Euler characteristic can have a polyhedral immersion (tight or otherwise) with 2 -fold rotational symmetry. This exhausts all possible symmetries, and thus completes the proof of the main theorem.

The fact that there are no symmetric tight immersions for the projective plane with one handle again makes this surface unique, in light of the following theorem proven in Section 3:

Theorem 1.2. Every orientable surface, and every non-orientable surface with Euler characteristic strictly less than -1, has a symmetric tight immersion in three-space.

The three surfaces that are excluded are the Klein bottle and the real projective plane, for which no tight immersion is possible, and the real projective plane with one handle, which is excluded by the previous theorem. All other surfaces have symmetric tight immersions, and frequently several different ones. Thus the projective plane with one handle continues to play a role as an important example of a tight surface.

\section{Definitions and basic results}

Given an abstract polyhedral surface $M$, a polyhedral map of $M$ is a function $f: M \rightarrow \mathbf{R}^{3}$ that maps the faces and edges of $M$ into linear subspaces of $\mathbf{R}^{3}$ (i.e., as planar triangles with straight edges). We assume that $f$ is nondegenerate, meaning that it does not reduce the dimension of any face or edge of $M$. The star of a vertex, $v$, is the union of the faces and edges that contain $v$.

A polyhedral mapping $f: M \rightarrow \mathbf{R}^{3}$ is an embedding if it is a one-to-one map. It is an immersion if it is locally one-to-one; that is, for every point $p$ of $M$, there is a neighborhood $U_{p}$ of $p$ where the restriction of $f$ to $U_{p}$ is one-toone. (For smooth surfaces, there are additional requirements that guarantee the existence of a tangent plane at every point, but these are not necessary in the polyhedral case.) The interiors of faces are always immersed, and the interiors of edges are immersed provided the adjacent faces don't overlap, 
so $f$ is an immersion provided the vertices are immersed. In a polyhedral map, a small neighborhood of a vertex is effectively the same as the star of the vertex, so we have the following lemma:

Lemma 2.1. A polyhedral map $f: M \rightarrow \mathbf{R}^{3}$ is an immersion if, and only if, the star of every vertex of $M$ is embedded by $f$.

A mapping $f: M \rightarrow \mathbf{R}^{3}$ is said to be tight provided that the preimage of every half-space of $\mathbf{R}^{3}$ is connected in $M$; that is, every plane cuts the image of $M$ into at most two pieces. This is also called the two-piece property. Several other interpretations of tightness can be found in the literature, e.g., [3]. Tightness is a property of the mapping $f$, not the surface itself, but it is common to speak of $M$ in place of $f(M)$ and let the mapping be implied. In practice, this ambiguity is resolved naturally by the context.

For polyhedral surfaces, tightness can be characterized as follows: A vertex $v$ of $M$ is a local extreme vertex if $f(v)$ is a vertex of the convex hull of the image of the star of $v$ (i.e., it is an isolated local maximum for the height function on $f(M)$ in some direction). A vertex is a (global) extreme vertex if its image is a vertex of the convex hull of $f(M)$. Note that $v$ will not be an extreme vertex (local or global) if it lies in the interior of the convex hull of some subset of its adjacent vertices; for example, if $v$ lies on the line segment between two of its neighbors, then $v$ can not be locally or globally extreme. With these definitions, we can state:

Lemma 2.2. A polyhedral map $f: M \rightarrow \mathbf{R}^{3}$ of a closed, compact, connected surface $M$ is tight if, and only if:

i) Every local extreme vertex is a global extreme vertex,

ii) every edge of the convex hull of $f(M)$ is contained in $f(M)$, and

iii) every vertex of the convex hull of $f(M)$ is the image of a single vertex of $M$.

This lemma can be found in the literature ([3] or [11], for example) as a result for embedded surfaces, without the third condition. See [6] for an example of why this condition is needed for immersions.

The intersection of a face of the convex hull of $f(M)$ with $f(M)$ is called a top set of $M$. (This is sufficient for our needs, but top sets are more general than this; see [3].) The lemma tells us that in a tight immersion, every edge of the convex hull is in the image of $M$, so the inverse image of the boundary of a face of the convex hull is a cycle in $M$. If this cycle does not separate $M$, then the cycle (or its image) is called a top cycle of $M$ (or $f(M)$ ).

The number of top cycles in a tight immersion of a surface is related to its Euler characteristic, $\chi(M)$. For a sphere, there are none; for other surfaces suppose $\alpha(M)$ is the number of top cycles in $M$. Then $2 \leq \alpha(M) \leq 2-\chi(M)$ when $\chi(M)$ is even, and $2 \leq \alpha(M) \leq 1-\chi(M)$ when $\chi(M)$ is odd. See [4] for further details. 
Note that if the boundary, $B$, of a face of the convex hull of $f(M)$ is not a top cycle, then $B$ separates $M$. In this case, one of the two regions in $M$ maps onto the complete face of the convex hull; if it didn't, then the height function in the direction perpendicular to the face (and toward the rest of the surface) would contain two maxima (one for each of the two regions of $M$ ). So moving the plane slightly in this direction would cut the surface into three pieces (one for each maximum, and a third containing $B$ ), contradicting the fact that the immersion is tight. So any face whose boundary is not a top cycle is entirely contained in $f(M)$. From a topological standpoint, this means that the intersection of $f(M)$ with its convex envelope is a sphere minus $\alpha(M)$ planar convex disks.

By a symmetry of $M$, we mean a rigid motion of $\mathbf{R}^{3}$ that carries $f(M)$ onto itself. For compact surfaces, such a motion will have to fix at least one point of $\mathbf{R}^{3}$, which we can take to be the origin. Any symmetry will be induced by one of three kinds of motions in $\mathbf{R}^{3}$ : A reflection across a plane, a rotation about a line, or a rotation-reflection (a rotation about a line followed by a reflection across a plane perpendicular to that line). These are distinguished by the fact that they fix a plane, a line, and a point, respectively.

If $M$ is a symmetric polyhedral surface, non-triangular faces can be subdivided while maintaining the symmetry, provided faces that are symmetric to each other are subdivided in the same way, and self-symmetric faces are divided so that the pieces preserve the underlying symmetry. Thus in the sections below, we can assume, without loss of generality, that $M$ is a triangulated surface.

\section{Symmetric tight immersions}

Closed surfaces can be organized into three families of related surfaces: Spheres with some number of handles (possibly zero), Klein bottles with some number of handles, and real projective planes with some number of handles. Kuiper's original proof ([13] and [14] ) that all but three surfaces have tight immersions involved showing that the sphere, the Klein bottle with a handle, and the real projective plane with two handles all have tight immersions, and that a handle can be added to a tight surface while maintaining tightness. We use a similar approach below to show that all these surfaces can be tightly immersed with symmetry.

For the orientable surfaces, we start with a sphere, or rather its polyhedral counterpart, a rectangular box. Drilling a tube through the box will add a handle to it, so drilling $n$ holes adds $n$ handles. If we place them equally spaced down the middle of the box, as in Figure 1, then the resulting surface will have three planes of reflective symmetry (parallel to the sides of the box) and three axes of 2 -fold rotational symmetry (through the centers of 
the sides of the box). Combining a rotation with the reflection in the plane perpendicular to the axis of rotation produces a rotation-reflection that is also a symmetry of the surface. Thus the sphere with $n$ handles can be made with many types of symmetries.

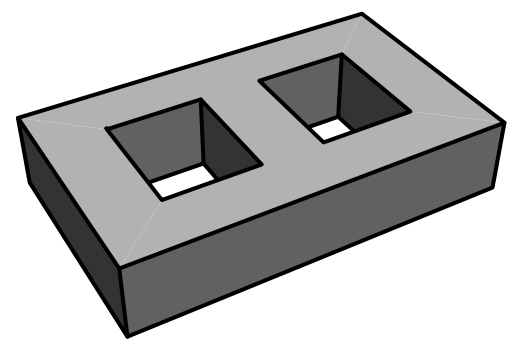

Figure 1. A tight torus with two handles can be made so that it has three planes of reflective symmetry and three axes of rotational symmetry. Any number of handles can be added in a similar way.

The tight Klein bottle with one handle originally described by Kuiper starts with a tight torus and adds a tube that connects the inside of the surface to the outside, as shown in Figure 2 (left). We can then add more handles as we did above. For an even number of additional handles, half can be placed on each side of the non-orientable handle to produce a tight surface with two planes of reflective symmetry and one axis of rotational symmetry. To make an odd number of handles, one more can be added "inside" the initial non-orientable handle (starting at the face through which the original handle passes and ending at the bottom face of the box). This retains the reflective and rotational symmetries.

Note, however, that this immersion does not have a reflection-rotation as a symmetry. We can obtain versions of the Klein bottle with two or more handles that do have such symmetries in the following way: Make the two attachment squares for the initial handle be of different sizes. Then for the next handle, make it in a symmetric position as shown in Figure 2 (right). This has the same three reflective symmetries and 2-fold rotational symmetries as the tori above, so it also has reflection-rotation symmetries. An even number of handles can be added by putting half on each side of the central handles, as before. An odd number can be added by putting the final one horizontally between the two side walls of the original box. In this way, a Klein bottle with at least two handles can be made with reflective, rotational and reflective-rotational symmetries.

Finally, consider the real projective plane. In [12], Kühnel and Pinkall provide a tight polyhedral immersion of the real projective plane with two 

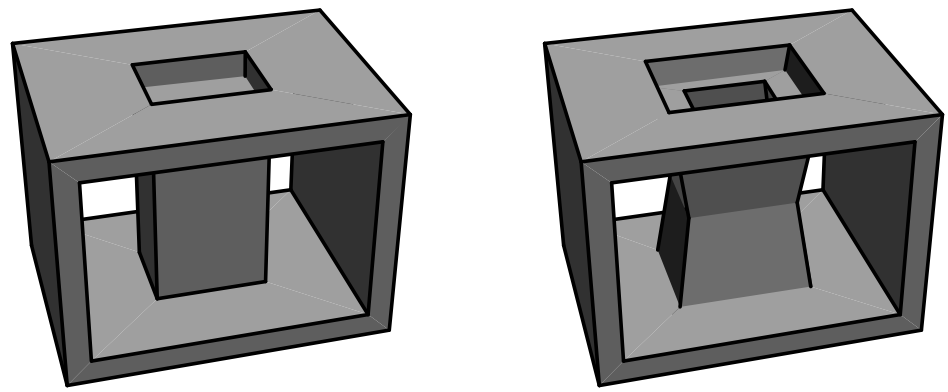

Figure 2. A tight Klein bottle with one handle (left) having one axis of rotational symmetry and two planes of reflective symmetry. After widening one end of the handle, a second handle can be added (right) that passes through the first, increasing the symmetries to include three axes of rotational and three planes of reflective symmetry. Self-intersection is indicated where two faces meet without a heavy black line.

handles that has 3-fold rotational symmetry, and they show how any number of handles can be added to this surface while maintaining the symmetry. Rotational and reflective-rotational symmetries are not possible for these surfaces, for the reasons discussed in the next section, so rotational symmetries are the only possibilities.

All the polyhedral surfaces provided in this section have smooth counterparts. A smoothing algorithm is given in [12] that can be applied to all these surfaces while preserving the symmetries, so Theorem 1.2 is true for both smooth and polyhedral surfaces.

\section{Symmetry and the projective plane with one handle}

Unlike the surfaces described above, which have both smooth and polyhedral tight immersions, the real projective plane has no smooth tight immersion [10] but does have polyhedral ones [8], so we need only consider polyhedral representations of this surface when proving Theorem 1.1.

Suppose that $M$ is a symmetric tight immersion of the projective plane with one handle (necessarily a polyhedral one). Then the symmetry is induced by a symmetry of $\mathbf{R}^{3}$, and must be either a rotation-reflection, a rotation, or a reflection, depending on whether it fixes a point, a line, or a plane in $\mathbf{R}^{3}$. Pinkall [16] classified all possible immersions of surfaces, up to image homotopy, and showed that for the projective plane, there are two distinct classes: Right- and left-handed versions of Boy's surface, which are mirror images of each other. Similarly, the projective plane with one handle has distinct left- and right-handed versions. Thus the mirror reflection of an immersion of the projective plane with one handle is in a different image 
homotopy class, and in particular, it is not identical to the original. This means no reflective symmetry is possible for that surface, and also rules out rotation-reflections, since these reverse the handedness as well. Thus the only possible symmetries for $M$ are rotations. We will eliminate these in the following sections. In 4.1, we show that no symmetries of order $n \geq 3$ are possible, and in 4.4 , we show there are no tight immersions having 2 -fold rotational symmetry.

\subsection{Rotations of order 3 or more. Suppose $M$ has an $n$-fold rotational} symmetry with $n \geq 3$. We begin by looking at the top cycles for the immersion. Cecil and Ryan [5] showed that the number of top cycles in a tight immersion is bounded by a formula dependent on the Euler characteristic of the surface. In particular, for a surface of odd Euler characteristic, if the number of top cycles is $\alpha$, then $2 \leq \alpha \leq 1-\chi(M)$. For the projective plane with one handle, this becomes $2 \leq \alpha \leq 1-(-1)=2$, so $\alpha=2$. Thus there are exactly two top cycles for $M$. Any symmetry must take a top cycle either to itself or to another top cycle. Since $n \geq 3$ in our case, each top cycle must map to itself, as there are only two of them. Thus each top cycle must itself be $n$-fold symmetric and must be perpendicular to the axis of rotational symmetry, which means that the two planes containing the top cycles are parallel. We can assume the axis of symmetry is the $z$ axis and the planes for the top cycles are at $z=-1$ and $z=1$.

The intersection of $M$ with its convex envelope is all of the envelope minus the interiors of the two faces bounded by the top cycles. Topologically, this is a cylinder. If we remove this cylinder, we are left with the interior portion of $M$. The Euler characteristic of the remainder is -1 since the Euler characteristic of the removed cylinder is 0 , and the sum of the values must be $\chi(M)=-1$. To close the surface again, we can add a topological disk to the two top cycles that form the boundary of the interior core. These can be formed by taking the cone over these curves to two new vertices, one above the upper top cycle at $(0,0,2)$ and one below the lower one at $(0,0,-2)$. Call this surface $\bar{M}$. Each disk has Euler characteristic 1, so $\chi(\bar{M})=-1+1+1=1$. As $\bar{M}$ is now a closed surface, this means it is a real projective plane. Since $M$ was contained between heights -1 and 1 , and since the top cycles are convex polygons, $\bar{M}$ is an immersion.

Consider the height function on $\bar{M}$ in the direction of the positive $z$ axis. Since the original surface is tight, the interior core has no local extreme vertices, hence the height function has exactly one maximum and one minimum (the two cone points). Since $\chi(\bar{M})=-1$, and the Euler characteristic equals the sum of the maxima and minima minus the saddles, this means there is one additional critical level between the maximum and minimum.

At this point, we would like to make the following argument: The critical level must contain a single saddle point, in order for the sum of the critical points to add up to the Euler characteristic. The critical level is a slice 
through the surface perpendicular to the axis of symmetry, so the level set at this height must also exhibit the $n$-fold rotational symmetry. Under this symmetry, a saddle point will have to map to another saddle, and since we have only one, it must map to itself. Thus the saddle point is on the axis of symmetry, and so the saddle itself must be $n$-fold symmetric. A simple saddle can exhibit at most a 2 -fold symmetry, however, since a small neighborhood of the point has two regions where the surface lies above the critical level, and a nontrivial symmetry would have to map these to each other. Our $n$ is at least 3, so this is a contradiction, and we should conclude that no $n$-fold symmetric tight immersion of $M$ exists for $n \geq 3$.

There is a problem with this argument, however: The claim that there is a critical "point" assumes a level of genericity that we are not guaranteed. It is possible for a saddle to occur along an edge or face contained in the critical level, for example (see Figure 3). Indeed, the critical levels of a polyhedral surface may be quite complex, and it may not be easy to localize the critical behavior to a single area of the level set. Furthermore, we can not simply say "put the vertices in general position" as this may not be possible while still maintaining the symmetry.
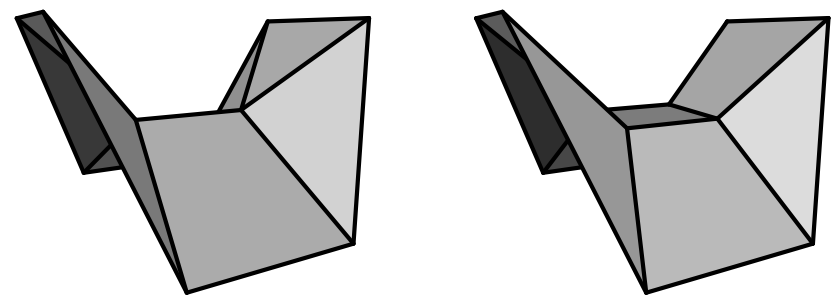

Figure 3. A saddle "point" can occur along an edge (left) or even at a face (right). If these are symmetric about the axis of 2 -fold rotation, we can not force the saddle to a vertex without destroying the symmetry.

A more sophisticated argument is needed in order to overcome these problems. The next section investigates the critical level more carefully, and shows that a level set with the desired properties can not be obtained.

4.2. The critical level in detail. Since there is only one minimum for the height function along the axis of symmetry, this means that the region below this critical level is a topological disk, and similarly for the region above. These disks each have Euler characteristic 1, so the Euler characteristic of the critical level must be -1 in order to have $\chi(\bar{M})=1$.

Let $C$ be the intersection of $\bar{M}$ with the plane perpendicular to the axis of symmetry at the critical height; i.e., $C$ is the critical level. By refining the triangulation of $\bar{M}$, if necessary, we can guarantee that the level set is made 
up of vertices, edges and faces of $\bar{M}$ (taking care to subdivide symmetric simplices in the same way). Since the level set is perpendicular to the axis of symmetry, $C$ must also show $n$-fold rotational symmetry, with the center of rotation at the point $P$ where the axis of rotation intersects the plane. If $P$ lies in the interior of any face or edge of $C$, we can subdivide it by placing a new vertex at $P$ and making edges to the vertices of the original simplex. This divides the simplex symmetrically.

In this way, we can assume that simplices of $C$ meet the axis of rotation only at vertices. In particular, this means no face or edge of $C$ is mapped to itself under the rotational symmetry, and also that each simplex (other than the vertices at $P$ ) is repeated $n$ times by the rotational symmetry about $P$. One consequence of this is, since the Euler characteristic is the number of vertices minus the number of edges plus the number of faces, the Euler characteristic of $C$ minus the number of vertices at $P$ is a multiple of $n$.

We now modify $C$ so as to remove any faces that lie in the level set, leaving only edges and vertices remaining in $C$. We will do this in such a way that symmetry is maintained, as well as the topology of $C$. That is, we will show:

Lemma 4.1. Any polyhedral immersion of the projective plane having $n$ fold rotational symmetry for $n \geq 3$ that is formed by two disks attached along a planar set $C$ of Euler characteristic -1 can be modified to produce a new immersion, again formed by two disks meeting at a planar set $\bar{C}$ having the same symmetry, but where $\bar{C}$ contains no faces.

The modification proceeds as follows: Suppose that $C$ contains a face. Then $C$ must contain at least one triangle with an edge that is not attached to another triangle in $C$ (otherwise every edge of every face in $C$ would be matched by another face in $C$, and so the triangles would form a closed surface completely contained in $C$; thus all of the projective plane would be in $C$, contradicting that it is formed by two disks meeting at $C$, one below and one above $C$ ). Let $T$ be such a triangle in $C$. Then at most two edges of $T$ are shared with other triangles of $C$, and at least one edge is part of the boundary of $C$. There are seven possible configurations for $T$, depending on how many edges meet other triangles of $C$, and on whether the remaining vertices of $T$ meet other edges or faces of $C$, as shown in Figure 4.

Each of these can be modified so as to remove the triangle from $C$. For (a), (b), (c), and (e), there is a vertex $v$ of $T$ that is not connected to other simplices of $C$, thus the rest of the star of $v$ must lie entirely on one side of the plane containing $C$. We can move $v$ slightly to this side of the plane, removing $T$ from $C$ but leaving the opposite edge of $T$ in $C$. For (d), (f) and $(\mathrm{g})$, triangle $T$ has at least one edge that is on the boundary of $C$, so the other face containing this edge is either below or above the plane of $C$; assume that is it below $C$. We can subdivide the edge (together with $T$ and 


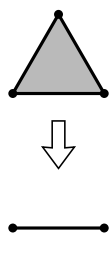

(a)

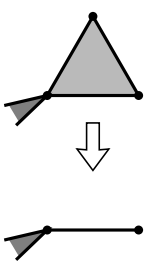

(b)

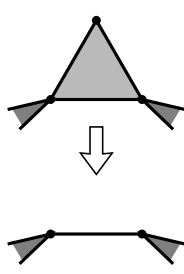

(c)

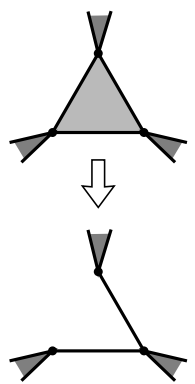

(d)

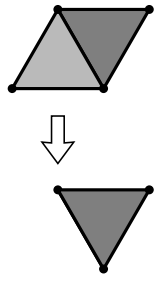

(e)

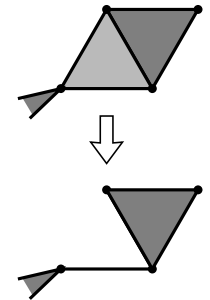

(f)

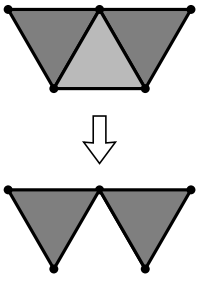

(g)

Figure 4. A triangle that is not entirely enclosed by other faces can be removed from the critical level either by moving a vertex out of the plane of the level set ( $a, b, c$ and e), or by subdividing an edge (and the two adjacent faces) and then moving the new vertex out of the plane ( $\mathrm{d}, \mathrm{f}$ and $\mathrm{g})$. The thin triangles at the corners represent connections to the rest of $C$ either by edges or faces.

its neighbor) and move the new vertex slightly downward. This removes $T$ from $C$, leaving the remaining two edges in $C$.

Note that the topology of the surface is not changed by these modifications, and so it is still formed by two disks meeting along (the modified) $C$. In each case the Euler characteristic is unchanged, so $\chi(C)$ continues to be -1 . Finally, provided that we modify every face that is symmetric to $T$ in the same fashion as we did $T$, the symmetry will be maintained. Note that since we changed $T$ only along edges where it did not meet $C$, and since no face is symmetric to itself by construction, the modifications for $T$ and its symmetric copies do not interfere with each other, so all can be modified simultaneously without trouble.

Performing this process on a triangle and its symmetric ones removes $n$ faces from $C$. If there are no more triangles in $C$, we are done; otherwise we go back and do it all again. Eventually, we will remove all the triangles from $C$, and this proves the lemma. 
In a similar fashion, if $C$ contains any edges with a vertex that is not incident to another edge, that vertex can be pulled to one side of the plane of $C$, removing that vertex and edge from $C$. This does not change the Euler characteristic, and if all the symmetric copies of the edge are modified in the same way simultaneously, the symmetry will be maintained. Again, this is possible to do since the symmetric copies will not interfere with each other. Repeatedly performing this operation yields a $\bar{C}$ such that every vertex meets either zero or at least two edges. But since $\bar{C}$ is attached to a disk (both below and above), and the boundary of a disk is a circle, and hence connected, $\bar{C}$ must also be connected, so an isolated vertex is not allowed. Thus every vertex of $\bar{C}$ meets at least two edges of $\bar{C}$.

We can assume, then, that for our surface $\bar{M}$, the critical level $C$ has the properties listed above; namely that it contains no faces, every vertex has at least two edges, it has $n$-fold rotational symmetry, and it is attached to a single topological disk above and another below. Note that our modifications to $\bar{M}$ may have introduced other critical points for the height function; but this is immaterial, as tightness is no longer an issue. The presence of such a set $C$ is all that we need.

We are now in a position to analyze the possible structures for $C$. It turns out that there is only one set $C$ with the proper symmetries and Euler characteristic, but that it can not be attached to two disks. Showing this will prove that the required immersion $\bar{M}$ of the projective plane does not exist. Hence our original tight immersion of the projective plane with one handle having $n$-fold rotational symmetry can not exist. This will complete the proof that there is no tight immersion of the projective plane with one handle having rotational symmetry of order $n \geq 3$.

4.3. The possible critical levels. Suppose we have decomposed $\bar{M}$ into two disks meeting at a critical level $C$, where $C$ has the properties described in the previous section: It contains no faces, every vertex meets at least two edges, it has $n$-fold rotational symmetry for $n \geq 3$ about a center of rotation, and $\chi(C)=-1$. If $C$ has no vertices on the center of rotation, then each edge and vertex of $C$ is repeated $n$ times by the symmetry, so $\chi(C)=k n$ for some $k \in \mathbf{Z}$. But since $n \geq 3, k n \neq-1$, a contradiction. Thus some vertices must lie on the center of rotation.

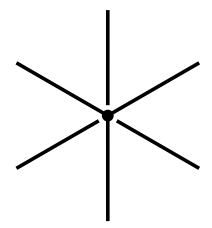

Figure 5. A configuration having 6-fold rotational symmetry that consists of two distinct vertices on the axis of rotation, each with three edges.

Every such vertex must be mapped by the rotation either to itself or some other vertex on the center of rotation. For example, if $n=6$, Figure 5 gives 
one possible arrangement. The orbit of a vertex must be some $v$ vertices where $v \mid n$. Each of these $v$ vertices is attached to the rest of $C$ by some number $e$ of edges, where $e \geq 2$ (since every vertex meets at least two edges), and so the total number of edges involved is ve. By symmetry, this must be divisible by $n$, i.e., $v e=k n$ for some $k \in \mathbf{Z}, k \geq 1$. In this way, the vertices on the axis can be broken down into a collection of $m$ distinct orbits, with $v_{i}$ vertices in the $i$-th orbit, each having $e_{i}$ edges such that $v_{i} e_{i}=k_{i} n$ for $i=1, \ldots, m$.

Suppose $\boldsymbol{p}$ is a vertex at the center of rotation, and $\boldsymbol{p} \boldsymbol{p}_{1}$ is an edge of $C$. Then since every vertex meets at least two edges, there is at least one more edge containing $\boldsymbol{p}_{1}$. If there is exactly one, then let $\boldsymbol{p}_{1} \boldsymbol{p}_{2}$ be this edge. We can continue to move along edges in this way until we come to an edge $\boldsymbol{p}_{i-1} \boldsymbol{p}_{i}$ where one of two things happen: Either $\boldsymbol{p}_{i}$ has more than one other edge (so there is no obvious choice for continuing our path), or $\boldsymbol{p}_{i}$ is on the center of rotation (so we have returned to the center, but not necessarily to the same vertex where we started). Call the first type of path a terminal path and the second a returning path. Each edge attached to a vertex on the center of rotation is part of either a terminal or a returning path.

Let $\bar{C}$ be the portion of $C$ consisting of the vertices on the center of rotation together with the terminal and returning paths (not including the final vertex of the terminal paths); see Figure 6 . Since $C$ has $n$-fold rotational symmetry, so does $\bar{C}$.
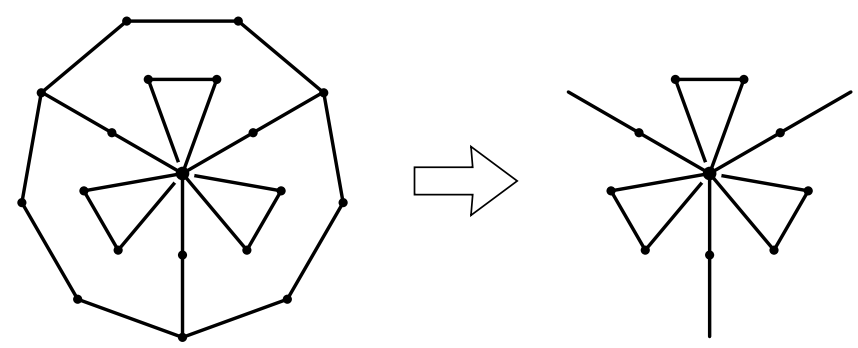

Figure 6. An example critical level (left) having 3-fold rotational symmetry. Here, there are two vertices at the center, so $m=2, v_{1}=1, e_{1}=6, k_{1}=2, v_{2}=1, e_{2}=3, k_{2}=1$ and $n=3$. The portion that becomes $\bar{C}$ is shown at the right. There are three terminal paths and three returning paths.

Note that since the terminal paths end at vertices having at least two other edges, each vertex of $C-\bar{C}$ still has at least two edges, and it still is connected. So if $V$ is the number of vertices of $C-\bar{C}$ and $E$ the number of edges, this means $E \geq V$, so $\chi(C-\bar{C})=V-E \leq 0$. Since $C$ and $\bar{C}$ have $n$-fold rotational symmetry, so does $C-\bar{C}$. Since $C-\bar{C}$ has no vertices on 
the center of rotation by construction, every edge and vertex is repeated $n$ times, so $\chi(C-\bar{C})=q n$ for some $q \in \mathbf{Z}$ with $q \leq 0$.

Let $v=\sum v_{i}$ be the total number of vertices at the center of rotation, and let $e=\sum v_{i} e_{i}=\sum k_{i} n=n \sum k_{i}$ be the total number of edges meeting the vertices at the center. Let $k=\sum k_{i}$, so $e=n k$. If we ignore the vertices on the center, each path (either returning or terminal) contains one more edge than vertex, so contributes -1 to $\chi(\bar{C})$. How many such paths are there? Let $p$ be the number of returning paths; then since each returning path accounts for two edges at the axis, the total number of paths is $e-p$. Thus $\chi(\bar{C})=v-(e-p)=v-e+p$.

Note that since each $e_{i} \geq 2$ and $v_{i} e_{i}=n k_{i}$, this means $v_{i} \leq n k_{i} / 2$, so $v=\sum v_{i} \leq(n / 2) \sum k_{i}=n k / 2=e / 2$. Thus $v \leq e / 2$. Also note that since each returning path uses two edges at the center, $p \leq e / 2$. So $v-e+p \leq e / 2-e+e / 2=0$, so $\chi(\bar{C}) \leq 0$.

We already know $\chi(C-\bar{C}) \leq 0$ and in fact $\chi(C-\bar{C})=q n$ for some integer $q \leq 0$. We also know $\chi(C)-\chi(\bar{C})=\chi(C-\bar{C})$ by construction. If $q \neq 0$, then $q \leq-1$ so $\chi(C-\bar{C}) \leq-n$, so $-1-\chi(\bar{C}) \leq-n$, or $\chi(\bar{C}) \geq n-1 \geq 2$ since $n \geq 3$. This is a contradiction since $\chi(\bar{C}) \leq 0$ from above. Thus it must be that $q=0$, so $\chi(C-\bar{C})=0$. This means $\chi(C)=\chi(\bar{C})$, so $\chi(\bar{C})=-1$. That is, $v-e+p=-1$. Now we saw above that $v \leq e / 2$, so $e / 2-e+p \geq-1$, or $p \geq e / 2-1$. On the other hand, $p \leq e / 2$ so $e / 2-1 \leq p \leq e / 2$.

If $e$ is odd, then this means $p=(e-1) / 2$ as the only possibility. Now the number of edges that lead to terminal paths is $e-2 p$, so all but one edge is used in returning paths; but this one edge must be matched by symmetry with $n-1 \geq 2$ other such edges, a contradiction.

So $e$ must be even, and either $p=e / 2$ or $p=e / 2-1$. It can't be the latter, as this would mean that all the edges but two are used in returning paths. Again, by the $n$-fold symmetry, if there is one, there must be at least $n \geq 3$ of these, a contradiction. So $p=e / 2$, and every edge is used in a returning path. Since the returning paths do not connect to any edges not in $\bar{C}$, and since $C$ itself is connected, this means $\bar{C}=C$; i.e., $\bar{C}$ is the entire level set.

Suppose $e_{i}=2$ for some $i$. A returning path starting at one of these can't come back to such a vertex, as this would form a closed loop, which would disconnect $C$, a contradiction. So we can think of the two returning paths through this point as one long returning path. Removing such a vertex from $v$ also removes two edges from $e$ while reducing $p$ by 1 . Thus $v-e+p$ will remain unchanged if we make this adjustment.

We can assume, without loss of generality, that the $e_{i}$ equal to 2 are all listed last; i.e., for some $\bar{m} \leq m, e_{i} \geq 3$ for $i \leq \bar{m}$, and $e_{i}=2$ for $i>\bar{m}$. Let $\bar{v}=\sum_{i=1}^{\bar{m}} v_{i}, \bar{k}=\sum_{i=1}^{\bar{m}} k_{i}, \bar{e}=\sum_{i=1}^{\bar{m}} v_{i} e_{i}=\sum_{i=1}^{\bar{m}} n k_{i}=n \bar{k}$, and let $\bar{p}=\bar{e} / 2$. Then the observations above indicate that $\bar{v}-\bar{e}+\bar{p}=v-e+p=-1$. But since $e_{i} \geq 3$ for $i \leq \bar{m}, v_{i} e_{i}=n k_{i}$ means $v_{i} \leq n k_{i} / 3$, so $\bar{v} \leq n \bar{k} / 3=\bar{e} / 3$. So 
$-1=\bar{v}-\bar{e}+\bar{p} \leq \bar{e} / 3-\bar{e}+\bar{e} / 2=-\bar{e} / 6$; i.e., $\bar{e} \leq 6$, and so $n \bar{k} \leq 6$. Since $n \geq 3$, this means $\bar{k} \leq 2$. If $\bar{k}=1$, then $\bar{e}=n \bar{k}=n$ means $\bar{p}=\bar{e} / 2=n / 2$; but by symmetry, each path maps to another path, hence $p \geq n$, a contradiction. So $\bar{k}=2$. This means $6 \geq n \bar{k}=2 n \geq 2 \cdot 3=6$, so $2 n=6$, and hence $n=3$.

So we know $n=3, \bar{k}=2, \bar{e}=6$ and $\bar{p}=3$. Then $\bar{v}-\bar{e}+\bar{p}=-1$ implies $\bar{v}=2$. Since $n=3$ and there are only two vertices at the center of rotation, each vertex must map to itself (as there can't be an orbit of length three). So $\bar{m}=2$, and $v_{1}=1, v_{2}=1$. Since $2=\bar{k}=k_{1}+k_{2}$, this means $k_{i}=1$, so from $v_{i} e_{i}=n k_{i}$, we conclude $e_{i}=3$. Thus there are two vertices each with three edges (as in Figure 5) connected by three returning paths. A returning path can not return to the same vertex, since the vertex must exhibit 3 -fold symmetry, so the returning paths at each edge of one vertex must lead to the other vertex. Thus there is only one possible configuration for $\bar{C}$ (up to topological type), as seen in Figure 7 (left).
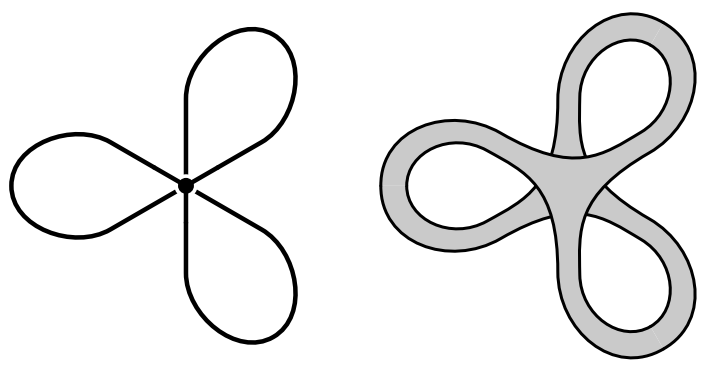

Figure 7. The only possible critical set has 3-fold symmetry, two vertices on the axis of rotation, each with three edges, and three returning paths (left). By "fattening" the edges, we see that this arrangement has only one boundary curve, so it can't be connected to two disks to form a projective plane; in fact, it is a torus minus a disk.

Recall that $C=\bar{C}$, and $C$ is a level set that is attached to a disk above $C$ and another disk below $C$. Note, however, that this configuration can not be attached to two disks. One way to see this is to widen the paths slightly and note that the result has only one boundary curve (see Figure 7, right), so can be attached to only one disk.

Since this was the only possible symmetric level set with Euler characteristic -1 , and it can't attach to the disks above and below, this proves that there is no tight immersion of the projective plane with one handle having $n$-fold rotational symmetry for $n \geq 3$. Thus we have eliminated all possibilities except 2-fold rotations.

4.4. Rotations of order 2. The previous sections considered the case of an $n$-fold rotational symmetry for $n \geq 3$ and showed that no tight immersion 
of the projective plane with one handle can be formed with such a symmetry. This leaves the case for $n=2$ to be considered. We will address this last situation by proving the following, more general, result:

Lemma 4.2. There is no immersion (tight or otherwise) of a closed, compact polyhedral surface with odd Euler characteristic that has a rotational symmetry of order 2 .

While our proof is for polyhedral surfaces, the technique should carry over to the smooth case as well. Once we have proved this lemma, we will have completed our proof of Theorem 1.1, since the real projective plane with one handle has odd Euler characteristic and we have already eliminated all other possible symmetries.

The proof of this lemma rests on two facts. The first is a result of Banchoff [2] that the number of triple points in an immersion of a surface in space is equivalent, modulo 2, to the Euler characteristic of the surface. This means that a surface of odd Euler characteristic has an odd number of triple points (and in particular, has at least one). The second is the following: In an immersion of a triangulated surface with 2 -fold rotational symmetry, no triangle is self-symmetric; that is, the symmetry takes each triangle to a different one.

To see this, suppose some triangle $a b c$ is mapped to itself by the symmetry. Then the rotation must interchange two vertices, say $a$ and $b$, and leave the other one, $c$, fixed. Then $c$ lies on the axis of symmetry, and so does the midpoint of $a$ and $b$, which means that the axis of symmetry passes through the plane of $a b c$. Consider the slices of the surface by planes perpendicular to the axis of symmetry. Note that each slice inherits the 2 -fold rotational symmetry, and that the slices that pass through $a b c$ contain self-symmetric edges that intersect the center of rotation for the slice.

As we pass through the various slices, the level curves change continuously except at critical levels. Since these slices must also maintain the 2-fold symmetry, a portion of a level curve that is a self-symmetric section must remain self-symmetric in nearby levels, and hence must continue to pass through the axis of rotation. This can change at a critical level, but only if another portion of the level set comes in contact with the self-symmetric curve. By the 2-fold symmetry, however, any contacts must come in pairs, and so an odd number of level curves must come together at such a point. After passing the critical level, there will still be an odd number of level curves, so one of these still must be self-symmetric, hence it must still pass through the axis of symmetry.

Thus there is no way to eliminate a segment that passes through the axis. Since the surface is compact, high enough level sets must be empty (every level curve will shrink to a point and disappear at a maximum), but since a segment of a level curve passing through the axis can't be removed, no 
level set can contain such a segment. This contradicts the fact that we must have such a segment when there is a self-symmetric triangle, hence there is no such triangle.

One last subtlety must be considered, however: Suppose there were several self-symmetric segments passing through the axis. Could these be used to eliminate each other? For example, could two such segments combine at a critical point to form two curves that do not pass through the axis? The answer is "yes, but not in an immersion". Such a configuration is shown in Figure 8, but any such arrangement would require that the self-symmetric segments would meet at a vertex on the axis, and the star of this vertex would contain self-intersection, and so this vertex star would not be embedded, hence the surface would not be immersed.

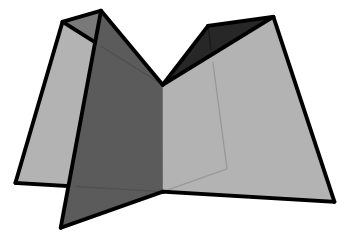

Figure 8. Two self-symmetric level curves (the two lines crossing at the bottom) combine to form a symmetric pair (the two separated lines at the top); but in doing so, they create a Whitney umbrella, where the surface is not immersed.

A similar argument shows that no self-symmetric edge can lie entirely on the axis of rotation, since then the two faces meeting at that edge would be paired by the symmetry; slices perpendicular to the axis of rotation through this pair would form self-symmetric level curves like the ones in the previous proof, but we have just seen that such curves are not allowed.

With these results in hand, we can now prove Lemma 4.2. Suppose $M$ is an immersion of a polyhedral surface of odd Euler characteristic, and suppose that $M$ has 2-fold rotational symmetry. Note that Banchoff's result tells us that there are an odd number of triple points. The symmetry must map each triple point to another triple point (either itself or a different one), so any triple point not mapped to itself is paired with a second one by the symmetry. This accounts for an even number of triple points, hence there must be at least one that is self-symmetric. This means it lies on the axis of rotation, and so a neighborhood of this triple point must show 2 -fold rotational symmetry.

A triple point is formed by three faces intersecting, and so, to have 2fold symmetry, the rotation must interchange two of these faces and map the third to itself. We have already shown above that no triangle can be self-symmetric in an immersion, so this is a contradiction. Thus $M$ can not have 2-fold rotational symmetry after all.

At first glance, this argument seems reasonable, but it has a problem as it currently stands. It assumes that the triple points are generic, that is, 
that they fall in the interior of faces, and occur where exactly three faces meet. This need not be the case, however; for example, one could have four faces meeting at a common point arranged so as to have 2 -fold rotational symmetry (see Figure 9). Such a "quadruple point" actually represents several triple points combined at one location. In order for Banchoff's theorem to count them properly, this degenerate configuration must be perturbed to break up the self-intersection into generic triple points.
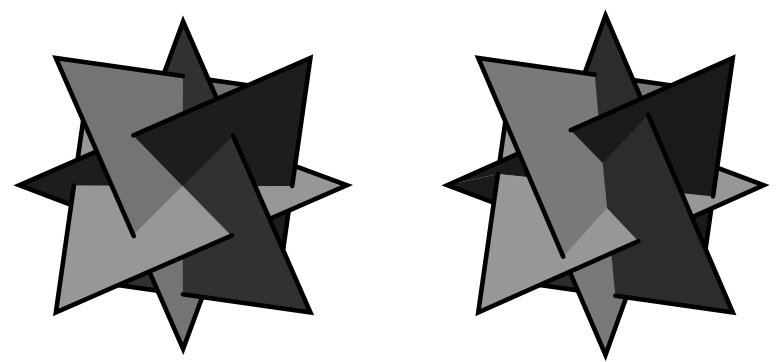

Figure 9. Four faces can meet at a single point (left) with 2 -fold (actually 4-fold) rotational symmetry about an axis vertical to the page. Moving the top and bottom vertices toward the viewer and the left and right ones away (right), we maintain the 2-fold symmetry and break up the quadruple point into four triple points; two are visible here.

The original point has six lines of self-intersection meeting at the quadruple point (one for each pair of the four faces). As we adjust two of the faces slightly (and symmetrically), these lines move apart, forming the edges of a tetrahedron whose vertices are the triple points where groups of three faces meet (there are four such groups of three since there are four faces in total). Thus this configuration actually represents four triple points, and there still must be a self-symmetric triple point elsewhere in the surface.

It would be tempting to say "put the surface into general position", but we want our modified surface to retain the original 2-fold symmetry, so this must be handled with some care. In the next section, we show how this can be done, and thus complete the proof.

4.5. Making the immersion generic. Our argument in the previous section relies on counting the number of triple points in the immersion, but there are a number of non-generic behaviors that can complicate this process. For example, if three faces meet along a common line (rather than at a point), there are an infinite number of triple points. As we have already seen (Figure 9), several triple points can be combined to form crossings of four or more faces. We need our triple points to occur in the interiors of faces where exactly three faces meet. 
In order to overcome these problems, we would like to move the surface into general position, but doing so might disrupt the symmetry. Instead, we need to look at the possible problem configurations and show that they can be removed while maintaining the 2 -fold rotational symmetry.

We have already noted that every face is paired by the symmetry with another (different) face of the triangulation. As we adjust one triangle, we need to make corresponding changes in the other in order to preserve the symmetry. A triangle and its pair intersect each other if, and only if, they meet the axis of symmetry. (If a triangle intersects the axis of symmetry, then that point is mapped to itself by the rotation so it also must be on the other triangle. If a triangle and its pair intersect at a point not on the axis, they also intersect at the point symmetric to this, and since the triangles are convex, they intersect all along the line segment between these two points. This line passes through the axis of symmetry, since it is a 2 -fold rotation).

If a triangle does not meet the axis of rotation, then it and its pair can be moved slightly (in symmetric ways) to remove any non-generic behavior from both triangles. Since the paired triangles don't intersect, these changes will not interfere with each other. Thus any unwanted configurations that are away from the axis can be removed while keeping the symmetry intact. So the only troublesome non-generic behavior occurs at the axis of symmetry. Since triangles that intersect the axis also intersect their pairs, these configurations always involve sets of symmetric pairs of faces.

There are two distinct kinds of pairs of faces: Those that share vertices and those that don't. If a face and its pair have a vertex or edge in common, then they don't intersect elsewhere, since the vertex stars are embedded in an immersion (Lemma 2.1). If they share a single vertex, that vertex must be on the axis of rotation, and it can be moved along the axis while maintaining the symmetry, thus removing it from any non-generic configuration.

If the faces share an edge, then the vertices of this edge map either to themselves or to each other. The first case is not possible, however, since if they map to themselves, then the edge lies along the axis of symmetry; but we saw in Section 4.4 that no self-symmetric edge lies on the axis. So if the faces share an edge, the vertices of the edge map to each other, and hence that edge must be perpendicular to the axis of rotation. We can move that edge parallel to the axis without disrupting the symmetry, thus removing the faces from the non-generic configuration.

In this way, we can guarantee that any problem area involves only symmetric pairs of intersecting faces that have no vertices in common. Such a pair can be made to intersect either along a segment in their interiors or not at all, as follows: If one of the vertices of the face lies on the axis, so does its counterpart in the symmetric triangle; both can be moved away from the axis while maintaining symmetry. If the faces no longer intersect, we are done. If an edge of one of the triangles intersects the axis, then so does 
it symmetric counterpart, and at the same point on the axis. In this case, symmetric vertices of each edge can be moved slightly (and symmetrically) so that the edges no longer intersect each other or the axis. Again, if the faces of the symmetric pair no longer meet, then we are done, otherwise they meet at a point on the axis that is in their interiors.

At this point, if the faces meet in their interiors, they do so either along a line segment in their interiors, or (if they lie in a common plane) in a 2dimensional region. In the latter case, any vertex and its pair can be moved so as to change the plane of the triangles slightly, causing the planes to intersect in a line segment or not at all. The planes of the paired triangles will change in opposite ways due to the symmetry, so they will not remain parallel after the adjustment, and so can't intersect in a 2-dimensional region.

Note that the line of intersection of two such faces is perpendicular to the axis of symmetry. To see this, we note that if the line of intersection were contained in the axis, then the two symmetric faces would have to lie in a common plane, and so would have a 2-dimensional overlap that would have been removed above. So some point on the line of intersection is not on the axis; but any point of intersection must have its corresponding point on the paired triangle, and since the symmetry is 2 -fold, this point lies directly opposite the original. Thus the line of intersection is the line between these two points, and so is perpendicular to the axis of symmetry.

Finally, note that moving a vertex of one of the faces together with its symmetric pair on the other will cause the line of intersection either to move parallel to that axis or to rotate around it (or both). The movements that cause the intersection to rotate in place are a set of measure zero, so the line of intersection always can be translated along the axis by a slight movement of vertices of the paired faces.

In this way, any non-generic behavior at the axis of symmetry can be broken up while still maintaining the symmetry. Thus the intersections within the surface can be made generic while still keeping the immersion a symmetric one, so we can guarantee that the triple points all occur in the interiors of faces where only three triangles meet. These are the conditions required by Banchoff's theorem, and so our arguments in Section 4.4 now can be made without difficulty, and we have proven Lemma 4.2.

This completes the proof of the main result, since the lemma shows that there is no tight immersion of the real projective plane with one handle having 2 -fold rotation, and Section 4.1 ruled out rotations of higher order.

\section{Conclusion}

In Section 3 we showed that the surfaces of even Euler characteristic all can be tightly immersed with multiple symmetries. We produced only rotations and rotation-reflections of order 2 , however. What higher order rotations 
and rotation-reflections are possible? Since top-cycles must be mapped to top-cycles, and these are bounded by the Euler characteristic, the number of handles certainly will play a role in the answer. This is addressed in [9].

For surfaces of odd Euler characteristic, Pinkall's classification of surfaces under image homotopy [16] can be used as in Section 4 to show that only rotational symmetries are possible. Arguments similar to those used in Lemma 4.2 should show that $n$-fold rotations are possible only for odd $n$. Again, however, the number of handles will control which odd rotations are possible. This issue also is considered in [9].

The real projective plane with one handle continues to be an unusual example among tight surfaces. It is the only one that has a polyhedral tight immersion but no smooth one, and now we see that it is the only surface that can be made tight but not symmetrically. Further study of this peculiar object may yield additional features that distinguish it from other surfaces.

\section{References}

[1] T.F. Banchoff, Tightly embedded 2-dimensional polyhedral manifolds, Amer. J. Math., 87 (1965), 462-472, MR 0178472 (31 \#2729), Zbl 0136.21005.

[2] — Triple points and surgery of immersed surfaces, Proc. Amer. Math. Soc., 46 (1974), 407-413, MR 0377897 (51 \#14066), Zbl 0309.57017.

[3] T.F. Banchoff and W. Kühnel, Tight submanifolds, smooth and polyhedral, in 'Tight and taut submanifolds', edited by T.C. Cecil and S.-S. Chern, Cambridge U. Press, 1997, 51-118, MR 1486870 (99d:53063), Zbl 0904.53003.

[4] T.E. Cecil and P.J. Ryan, On the number of top-cycles of a tight surface in 3-space, J. London Math. Soc., 30 (1984), 335-341, MR 0771428 (86e:5702), Zbl 0528.53046.

[5] _ Tight and Taut Immersions of Manifolds, Research Notes in Mathematics, 107, Pitman Publ., Boston, London, Melbourne, 1985, MR 0781126 (87b:53089), Zbl 0596.53002.

[6] D.P. Cervone, Tight immersions of simplicial surfaces in three space, Topology, 35 (1996), 863-873, MR 1404913 (97e:57026), Zbl 0858.53051.

[7] _ Tightness for smooth and polyhedral immersions of the real projective plane with one handle, in 'Tight and taut submanifolds', edited by T.E. Cecil and S.-S. Chern, Cambridge U. Press, 1997, 119-133, MR 1486871 (98j:53071), Zbl 0926.52018.

[8] _ A tight polyhedral immersion in three-space of the projective plane with one handle, Pacific J. Math., 196 (2000), 113-122, MR 1797237 (2001m:57038).

[9] _ On the possible symmetries of tight immersions of surfaces in three-space, in preparation.

[10] F. Haab, Immersions tendues de surfaces dans $\mathrm{E}^{3}$, Comment. Math. Helv., 67 (1992), 182-202, MR 1161280 (93e:53068), Zbl 0763.53060.

[11] W. Kühnel, Tight and 0-tight polyhedral embeddings of surfaces, Invent. Math., 58 (1980), 161-177, MR 0570878 (82h:53004), Zbl 0432.53041.

[12] W. Kühnel and U. Pinkall, Tight smoothing of some polyhedral surfaces, in 'Global Differential Geometry and Global Analysis' (Berlin 1984), edited by D. Ferus et al., 
Lecture Notes in Mathematics, 1156, Springer, Berlin, 1985, 227-239, MR 0824071 (87h:53085), Zbl 0566.53054.

[13] N.H. Kuiper, On surfaces in Euclidean three space, Bull. Soc. Math. Belg., 12 (1960), 5-22, MR 0123281 (23 \#A609), Zbl 0196.25901.

[14] _ Convex immersions of closed surfaces in $\mathrm{E}^{3}$, Comm. Math. Helv., 35 (1961), 85-92, MR 0124865 (23 \#A2175), Zbl 0243.53043.

[15] - There is no tight continuous immersion of the Klein bottle into $\mathbf{R}^{3}$. Preprint, IHES, 1983.

[16] U. Pinkall, Regular homotopy classes of immersed surfaces, Topology, 24 (1985), 421-434, MR 0816523 (87e:57028), Zbl 0583.570.

Received July 2, 2001 and revised April 13, 2002.

Department of Mathematics

Union COLlege

SCHENECTADY, NY 12308

E-mail address: dpvc@union.edu 